\title{
Analysis of the role of DAMTC in lung adenocarcinoma cells based on the DNA microarrays
}

\author{
BINLIANG WANG, YUANYUAN CAI, YIMING KONG, XIAOBO LI, \\ HAIWEI FU, SONG ZHANG and TIANWEI ZHANG \\ Respiratory Medicine Department, Taizhou First People's Hospital, \\ Taizhou, Zhejiang 318020, P.R. China
}

Received May 4, 2016; Accepted January 21, 2019

DOI: $10.3892 / \mathrm{ol} .2019 .10146$

\begin{abstract}
The present study aimed to investigate the effect of 7, 8-diacetoxy-4-methylcoumarin (DAMTC) on lung adenocarcinoma cells (A549) and analyze the molecular mechanism underlying DAMTC-treated lung adenocarcinoma. Gene expression profile GSE29698 was downloaded from the Gene Expression Omnibus database. The differentially expressed genes (DEGs) in 3 DAMTC-treated A549 samples were analyzed and compared with 3 DAMTC-untreated samples using the limma package. Gene Ontology (GO) and pathway enrichment analyses of DEGs were performed, followed by the functional annotation and protein-protein interaction (PPI) network construction. Finally, pathway crosstalk analysis was conducted. A total of 500 upregulated and 389 downregulated DEGs were identified. The upregulated and downregulated DEGs were enriched in different GO terms and pathways, including metabolic process, p53 signaling pathway and metabolic pathways. A total of 9 DEGs were determined to have node degrees $>16$ in the PPI network, including interleukin 6 (IL6), MDM2 oncogene, E3 ubiquitin protein ligase (MDM2), cell division cycle 42 (CDC42) and MYC associated factor $\mathrm{X}(M A X)$. Furthermore, numerous DEGs were identified to function as transcription factors and tumor suppressor genes, including histone deacetylase 3 and $M A X$. Additionally, apoptosis, tight junction, and endocytosis pathway were determined to cross-talk with small cell and non-small cell lung cancer. The DEGs (IL6, MDM2, CDC42 and $M A X)$ involved in different pathways, including the p53 signaling pathway and endocytosis, may be the potential targets for DAMTC in lung adenocarcinoma. The elucidation of the underlying mechanism of the DAMTC effect may make it a potential drug.
\end{abstract}

Correspondence to: Dr Binliang Wang, Respiratory Medicine Department, Taizhou First People's Hospital, 218 Hengjie Road, Taizhou, Zhejiang 318020, P.R. China

E-mail: 1060342144@qq.com

Key words: lung adenocarcinoma, differentially-expressed genes, protein-protein interaction network, pathway crosstalk analysis

\section{Introduction}

Lung cancer remains the leading cause of cancer-associated mortality, annually resulting in $>1,000,000$ mortalities globally in 2012 (1). Additionally, 1,200,000 new cases are diagnosed every year and prognoses of lung cancer are poor, as estimated worldwide in 2012 (2). Lung adenocarcinoma is the predominant histological subtype of non-small lung cancer (3). The mean 5-year survival rate of lung adenocarcinoma is $<15 \%$ in the urban areas of China as estimated in 2015, primarily due to the late-stage detection and a paucity of late-stage treatments (4). The availability of effective anticancer drugs is considered to be the key for improvement in the treatment of lung adenocarcinoma.

7, 8-Diacetoxy-4-methylcoumarin (DAMTC) is a thioderivative of 4-methyl coumarin (5). Coumarins belong to the flavonoid class of plant secondary metabolite, which have been demonstrated to exhibit diverse and beneficial biological activities, including antitumoral, anticoagulant and anti-inflammatory properties (5). Natural coumarins or synthetic analogs have attracted intense interest for their applicability as drugs (6). They have been determined to have a variety of therapeutic applications, including antitumor and anti-human immunodeficiency virus therapy (7), and antioxidant (8) and antibacterial (9) applications. Lacy and O'Kennedy (10) demonstrated that genistein and esculetin could exert the most potent inhibitory effect on cell growth of two cell lines, A549, a lung carcinoma cell line, and MCF-7, a breast carcinoma cell line. Previously, the coumarin derivative DAMTC was indicated to inhibit cellular proliferation and induce apoptosis on human non-small cell lung cancer A549 cells (11). Furthermore, the observations of Goel et al (12) reported that upregulation of the nuclear factor- $\kappa \mathrm{B}, \mathrm{p} 53$ and Akt pathways, and downregulation of the mitogen activated protein kinase (MAPK) and Cox-2 pathways were involved in the molecular mechanism of apoptosis induction by DAMTC in A549 cells. However, the mechanisms of the anti-proliferative effects of DAMTC in lung adenocarcinoma are incompletely defined, and further insights into the mechanisms are required.

Previously, Goel et al (1) used the integrated proteomics and transcriptomics approach, and identified that DAMTC could regulate cell motility and cytoskeletal reorganization in lung adenocarcinoma. In the present study, 
differentially-expressed genes (DEGs) were identified in DAMTC-treated lung adenocarcinoma, compared with DAMTC-untreated controls, using the same gene expression profile. Comprehensive bioinformatics were used to analyze the significant pathways and functions, and to construct the protein-protein interaction (PPI) network, to determine the critical DEGs. Furthermore, the putative interactions between signaling pathways were analyzed. The present study aimed to investigate the potential molecular mechanism underlying DAMTC-induced apoptosis and inhibition of cell motility in lung adenocarcinoma.

\section{Materials and methods}

Microarray data and data preprocessing. The gene expression profile of GSE29698, deposited by Goel et al (1), was downloaded from the Gene Expression Omnibus database in National Center for Biotechnology Information (http://www.ncbi.nlm. nih.gov/geo/) based on the platform of GPL6884 Illumina HumanWG- 6 v3.0 expression beadchip. A total of 6 specimens were applied, including 3 specimens of DAMTC-treated lung adenocarcinoma cells (A549) and another 3 specimens of DAMTC-untreated A549 cell lines as controls.

The gene expression profile data were preprocessed using the limma (13) package in Bioconductor. Following background correction, quantile normalization and probe summarization, the gene expression matrix of specimens was received.

DEGs screening. Unpaired Student's t-test (13) in limma package was used to identify the DEGs in the DAMTC-treated A549 cell group, compared with the control group. False discovery rate (FDR) (14) was performed for multiple testing correction using the Benjamini and Hochberg method (15). The threshold for the DEGs was set as FDR $<0.01$ and $\log _{2}$ FC (fold change) $l \geq 2$.

Functional and pathway enrichment analysis of DEGs. Gene Ontology (GO) (16) categories, including biological process (BP), molecular function (MF) and cellular component (CC), of the selected DEGs were enriched from GO databases using Database for Annotation Visualization and Integrated Discovery (DAVID) (17). Additionally, the pathways of selected DEGs were enriched using DAVID from Kyoto Encyclopedia of Genes and Genomes (KEGG) (18) analysis. $\mathrm{P}<0.05$, as determined by the hypergeometric test (19), was selected as the threshold.

Functional annotation ofDEGs.Identification of tumor-associated genes (TAGs) and understanding their functions can be critical for investigating the roles of genes involved in tumorigenesis. The tumor suppressor gene (TSGene) database (http://bioinfo. mc.vanderbilt.edu/TSGene/) is a comprehensive literature-based database that provides detailed annotations for each TSG. The TAG database (http://www.binfo.ncku.edu.tw/TAG/) is designed to utilize information from well-characterized oncogenes and tumor suppressor genes to accelerate cancer research. According to the data information regarding transcription factors (TFs) from the TRANSFAC database (20), functional enrichment of the DEGs for transcription regulation was assessed. Additionally, the selected DEGs were mapped into the TSGene and TAG database to extract the genes that had transcriptional functions or functioned as TAGs.

PPI network construction. The PPI network is represented by an undirected graph with nodes indicating the genes and edges indicating the mapped interactions of the proteins encoded by the genes (21). The PPI network of the selected genes was constructed by using data from the Retrieval of Interacting Genes (STRING) database, which is a comprehensive database containing functional associations between proteins that are experimentally derived, as well as associations predicted by comparative genomics and text mining (22). The interaction pairs with the PPI combined score $>0.4$ were selected in this network, which corresponded to a medium-confidence network (23).

Pathway crosstalk analysis. If more significant protein interactions are detected between two pathways, these two pathways are probable to influence or interact with each other (cross-talk). Understanding the crosstalk between pathways is momentous for understanding the function of cells and more complex systems (24). In the present study, the KEGG pathway data and protein interaction data based on the PPI network were combined to investigate pathway crosstalk in the DAMTC-treated A549 cells. The detail is included in the studies by Li et al (24) and Liu et al (25).

\section{Results}

Identification of DEGs. For the dataset GSE29698, a total of 889 DEGs were identified in DAMTC-treated A549 cell groups, compared with DAMTC-untreated controls, including 500 upregulated genes and 389 downregulated genes. The heat-map of the DEGs is depicted in Fig. 1. The results demonstrated that the DEGs expression pattern could significantly distinguish the DAMTC-treated A549 cell samples from controls.

GO and pathway enrichment analysis of the DEGs. Functional and pathway enrichment analysis indicated that upregulated and downregulated DEGs in DAMTC-treated A549 cell groups were significantly enriched in different $\mathrm{GO}$ terms and KEGG pathways (Tables I and II). GO analysis demonstrated enrichment in upregulated DEGs involved in BP, including transcription, DNA-dependent and regulation of cellular metabolic process. Significant CC ontology terms of upregulated DEGs were associated with intracellular part. The majority of significant MF ontology terms of upregulated DEGs were associated with binding function and enzyme activity, including DNA binding and phosphatase activity. Furthermore, significant BP ontology terms of downregulated DEGs were associated with tissue morphogenesis, and morphogenesis of an epithelium. CC ontology terms of downregulated DEGs were also associated with intracellular part. Significant MF ontology terms of downregulated DEGs were associated with organic cyclic compound binding, and RNA polymerase II transcription corepressor activity (Table I).

Additionally, upregulated DEGs were enriched in different pathways, including the 553 signaling pathway, and fructose and mannose metabolism pathway. Downregulated DEGs 


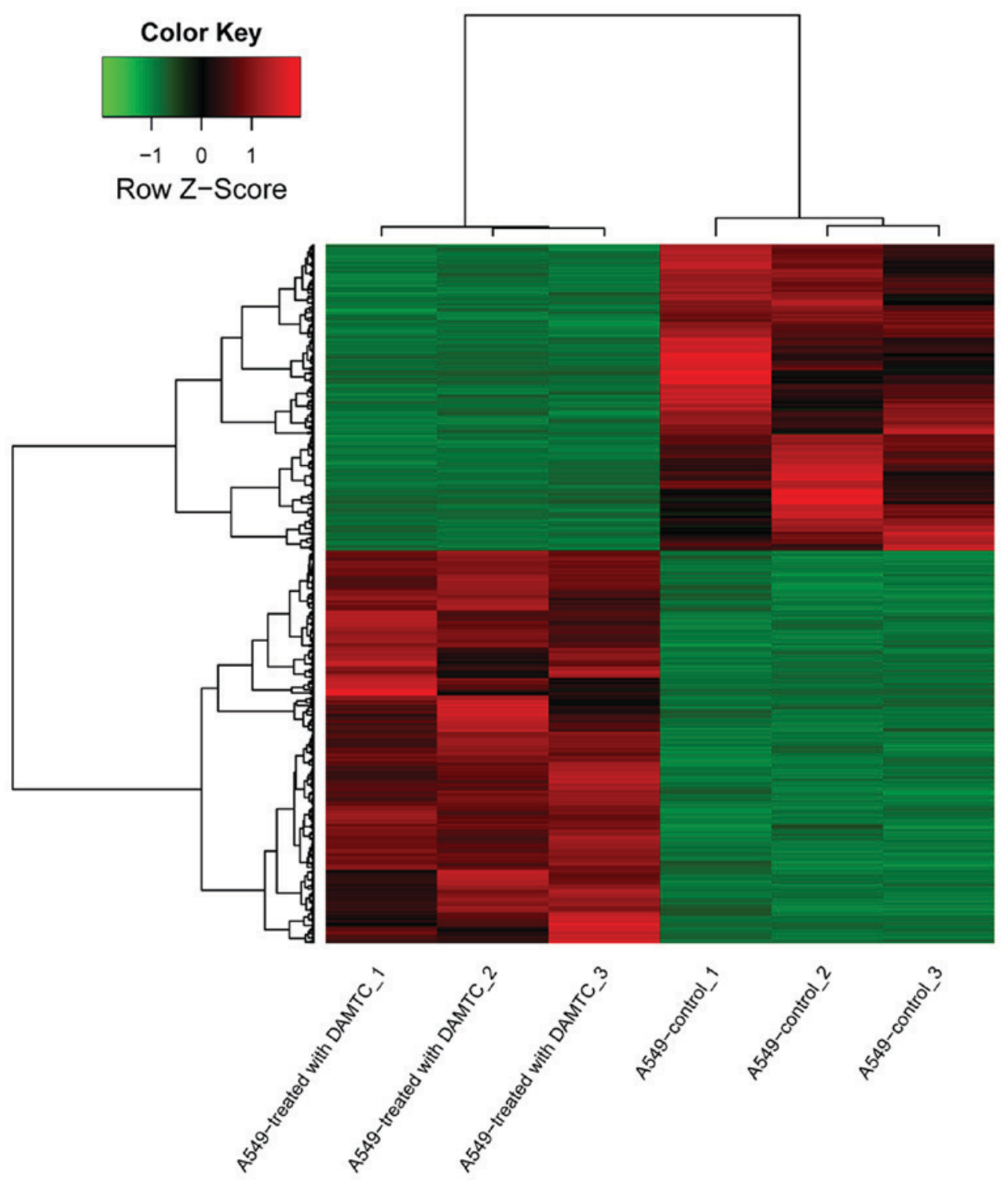

Figure 1. Heat-map of differentially-expressed genes. For each gene (row), red indicates a higher expression and green a reduced expression relative to the mean level of expression of the gene across the samples (columns), while black indicates genes not differentially expressed. DAMTC, 7,8-diacetoxy-4-methylcoumarin.

were also identified to be involved in different pathways, including ubiquitin mediated proteolysis, and metabolic pathways (Table II).

Function annotation of DEGs. The expression change of TFs, TSGs and other TAGs in DAMTC-treated A549 cell groups were observed (Table III). The upregulated functional genes included 34 TFs and 26 TSGs. Subsequently, MAX was identified to be a TF, while tumor protein p53 inducible nuclear protein 1 and bone morphogenetic protein 2 were identified to be TSGs. Additionally, histone deacetylase 3 (HDAC3) was identified as both a TF and a TSG. Additionally, the downregulated functional genes included $27 \mathrm{TFs}$ and 18 TSGs. Among them, sex determining region Y-box 4 and signal transducer and activator of transcription 1 (STATI) were identified to be TFs.

PPI network and the pathway crosstalk analysis. Based on the STRING database, the PPI network was constructed
(Fig. 2). The results demonstrated that a total of 9 genes had a node degree $>16$, including interleukin 6 (IL6; degree, 37), MDM2 oncogene, E3 ubiquitin protein ligase (MDM2; degree, 27), STAT1 (degree, 23), ataxia telangiectasia mutated (degree, 20), HDAC3 (degree, 19), cell division cycle 42 (CDC42; degree, 18), cytochrome c, somatic (degree, 17), bone morphogenetic protein 4 (degree, 17) and $M A X$ (degree, 17).

The result of pathway crosstalk analysis is depicted in Fig. 3 . The majority of the significant pathways were determined to have crosstalk with small cell lung cancer and non-small cell lung cancer. The apoptosis, tight junction and endocytosis pathways were determined to be cross-talking with small cell lung cancer and non-small cell lung cancer. Additionally, the bacterial invasion of epithelial cells, adherens junction, shigellosis and mechanistic target of rapamycin kinase signaling pathways had cross-talk with non-small cell lung cancer. In addition, small cell lung cancer and non-small cell lung cancer were also determined to have cross-talk. 
Table I. The enriched GO terms of differentially-expressed genes in 7,8-diacetoxy-4-methylcoumarin-treated lung cancer groups.

\begin{tabular}{|c|c|c|c|c|c|}
\hline Expression changes & Category & GO-ID & Name & Count & P-value \\
\hline \multirow[t]{15}{*}{ Upregulated } & \multirow[t]{5}{*}{ BP } & GO:0006351 & Transcription, DNA-dependent & 153 & $3.30 \times 10^{-11}$ \\
\hline & & GO:0031323 & Regulation of cellular metabolic process & 194 & $7.80 \times 10^{-11}$ \\
\hline & & GO:0060255 & Regulation of macromolecule metabolic process & 187 & $8.24 \times 10^{-11}$ \\
\hline & & GO:0051252 & Regulation of RNA metabolic process & 143 & $1.56 \times 10^{-10}$ \\
\hline & & GO:0019222 & Regulation of metabolic process & 207 & $2.08 \times 10^{-10}$ \\
\hline & \multirow[t]{5}{*}{$\mathrm{CC}$} & GO:0005622 & Intracellular & 387 & $2.45 \times 10^{-10}$ \\
\hline & & GO:0044424 & Intracellular part & 382 & $1.08 \times 10^{-9}$ \\
\hline & & GO:0005634 & Nucleus & 219 & $2.68 \times 10^{-9}$ \\
\hline & & GO:0043227 & Membrane-bounded organelle & 320 & $5.19 \times 10^{-9}$ \\
\hline & & GO:0043231 & Intracellular membrane-bounded organelle & 319 & $5.71 \times 10^{-9}$ \\
\hline & \multirow[t]{5}{*}{ MF } & GO:0003677 & DNA binding & 98 & $3.25 \times 10^{-5}$ \\
\hline & & GO:0016791 & Phosphatase activity & 18 & $4.00 \times 10^{-4}$ \\
\hline & & GO:0000988 & Protein binding transcription factor activity & 28 & $8.00 \times 10^{-4}$ \\
\hline & & GO:0005515 & Protein binding & 244 & $11.00 \times 10^{-3}$ \\
\hline & & GO:0005488 & Binding & 362 & $13.00 \times 10^{-3}$ \\
\hline \multirow[t]{15}{*}{ Downregulated } & \multirow[t]{5}{*}{ BP } & GO:0048729 & Tissue morphogenesis & 28 & $2.61 \times 10^{-6}$ \\
\hline & & GO:0002009 & Morphogenesis of an epithelium & 24 & $3.72 \times 10^{-6}$ \\
\hline & & GO:0044237 & Cellular metabolic process & 221 & $7.50 \times 10^{-5}$ \\
\hline & & GO:0044238 & Primary metabolic process & 224 & $8.50 \times 10^{-5}$ \\
\hline & & GO:0048736 & Appendage development & 12 & $8.77 \times 10^{-5}$ \\
\hline & \multirow[t]{5}{*}{$\mathrm{CC}$} & GO:0044424 & Intracellular part & 291 & $3.52 \times 10^{-8}$ \\
\hline & & GO:0043231 & Intracellular membrane-bounded organelle & 243 & $1.83 \times 10^{-7}$ \\
\hline & & GO:0005622 & Intracellular & 291 & $2.11 \times 10^{-7}$ \\
\hline & & GO:0043227 & Membrane-bounded organelle & 243 & $2.69 \times 10^{-7}$ \\
\hline & & GO:0043229 & Intracellular organelle & 257 & $2.01 \times 10^{-6}$ \\
\hline & \multirow[t]{5}{*}{ MF } & GO:0097159 & Organic cyclic compound binding & 154 & $2.31 \times 10^{-6}$ \\
\hline & & GO: 1901363 & Heterocyclic compound binding & 152 & $2.77 \times 10^{-6}$ \\
\hline & & GO:0001191 & $\begin{array}{l}\text { RNA polymerase II transcription factor binding } \\
\text { transcription factor activity involved in negative } \\
\text { regulation of transcription }\end{array}$ & 6 & $2.35 \times 10^{-5}$ \\
\hline & & GO:0001106 & RNA polymerase II transcription corepressor activity & 5 & $3.99 \times 10^{-5}$ \\
\hline & & GO:0003714 & Transcription corepressor activity & 14 & $8.22 \times 10^{-5}$ \\
\hline
\end{tabular}

GO, gene ontology; CC, cellular component; BP, biological process; MF, molecular function.

\section{Discussion}

The worldwide incidence rate of lung cancer is high (26). It is important to develop novel, more effective treatment modalities for dealing with this disease. DAMTC is a potential inducer of apoptosis and an inhibitor of cell growth (1). Thus, identifying the molecular mechanism underlying the effects of DAMTC is imperative. In the present study, microarray analysis demonstrated that 500 upregulated DEGs and 389 downregulated DEGs were identified in DAMTC-treated A549 cells, compared with DAMTC-untreated controls. Among the DEGs, IL6, which was downregulated, was a hub protein with the highest degree in the PPI network. The upregulated DEGs MDM2, CDC42 and MAX, which were also hub genes, had different functions and were involved in different pathways, including the p53 signaling and endocytosis pathways. Additionally, the apoptosis, tight junction, and endocytosis pathways were determined to cross-talk with small cell lung cancer and non-small cell lung cancer.

IL6 encodes a multifunctional cytokine, which can function in inflammation and the maturation of B cells (27). Hodge et al (28) demonstrated that the release of various survival factors, including IL6, serve to block apoptosis in cancer cells during the inflammatory process, keeping them alive in toxic environments. Additionally, studies have demonstrated that drugs designed to restore apoptosis exhibit potential to effectively treat numerous cancer types, including inhibitors of apoptosis, protein inhibitors and MDM2 antagonists (29,30). Furthermore, in keeping with the previous study, our study identified that IL6 was downregulated in DAMTC-treated A549 groups and was involved in the pathways in cancer (Table I). Thus, it was indicated that DAMTC may induce cancer cell apoptosis through targeting IL6 in lung adenocarcinoma. 
Table II. The enriched pathways of differentially-expressed genes in 7, 8-diacetoxy-4-methylcoumarin-treated lung cancer groups.

\begin{tabular}{|c|c|c|c|c|c|}
\hline $\begin{array}{l}\text { Expression } \\
\text { changes }\end{array}$ & KEGG-ID & Name & $\mathrm{n}$ & P-value & Genes \\
\hline \multirow[t]{8}{*}{ Upregulated } & 04115 & p53 signaling pathway & 9 & 0.0001 & $\begin{array}{l}\text { APAF1, CCNG2, CYCS, GADD } 45 A, \text { MDM2, } \\
\text { PPM1D, RPRM, SESN1 and SESN3 }\end{array}$ \\
\hline & 00051 & $\begin{array}{l}\text { Fructose and mannose } \\
\text { metabolism }\end{array}$ & 5 & 0.0032 & ALDOC, C12orf5, HK2, MTMR1 and PFKFB4 \\
\hline & 05219 & Bladder cancer & 5 & 0.0062 & FGFR3, MAP2K1, MDM2, PGF and RASSF1 \\
\hline & 04722 & $\begin{array}{l}\text { Neurotrophin signaling } \\
\text { pathway }\end{array}$ & 9 & 0.0095 & $\begin{array}{l}\text { CDC42, GAB1, MAP2K1, MAP3K3, MAPK7, } \\
\text { NFKBIE, NTF3, PDK1 and ZNF274 }\end{array}$ \\
\hline & 05200 & Pathways in cancer & 17 & 0.0103 & $\begin{array}{l}\text { BMP2, CCNA1, CDC42, CYCS, EGLN1, FGF18, } \\
\text { FGFR3, FZD1, FZD4, FZD7, MAP2K1, MAX, } \\
\text { MDM2, PGF, PIAS3, RASSF1 and RASSF5 }\end{array}$ \\
\hline & 04130 & $\begin{array}{l}\text { SNARE interactions in } \\
\text { vesicular transport }\end{array}$ & 4 & 0.0180 & BNIP1, STX11, STX7 and VAMP1 \\
\hline & 04144 & Endocytosis & 11 & 0.0265 & $\begin{array}{l}\text { CDC42, CXCR4, DNM2, FGFR3, GIT2, HLA-E, } \\
\text { MDM2, RAB11FIP1, SH3GL2, SH3GL3 and } \\
\text { VPS37B }\end{array}$ \\
\hline & 05211 & Renal cell carcinoma & 5 & 0.0472 & CDC42, EGLN1, GAB1, MAP2K1 and PGF \\
\hline \multirow[t]{7}{*}{ Downregulated } & 05217 & Basal cell carcinoma & 6 & 0.0008 & $\begin{array}{l}\text { BMP4, FZD2, GLI2, STK36, TCF7L2 and } \\
\text { WNT7B }\end{array}$ \\
\hline & 00130 & $\begin{array}{l}\text { Ubiquinone and other } \\
\text { terpenoid-quinone } \\
\text { biosynthesis }\end{array}$ & 2 & 0.0079 & COQ5 and COQ7 \\
\hline & 01100 & Metabolic pathways & 33 & 0.0130 & $\begin{array}{l}\text { ACSS1, AK2, ATP6V1E2, B3GALT6, BAAT, } \\
\text { BCAT1, COQ5, COQ7, CYP2R1, DPAGT1, } \\
\text { GGPS1, GPT2, HKDC1, HLCS, OXSM, PAFAH2, } \\
\text { PCK2, PFAS, PIGM, PIGV, PIGW, PIK3C2B, } \\
\text { PLD1, PMM2, POLG2, POLR1B, POLR3H, } \\
\text { PSPH, SEPHS2, SLC33A1, ST3GAL5, ST6GAL1 } \\
\text { and SYNJ2 }\end{array}$ \\
\hline & 00563 & $\begin{array}{l}\text { Glycosylphosphatidyli- } \\
\text { nositol-anchor biosynthesis }\end{array}$ & 3 & 0.0132 & PIGM, PIGV and PIGW \\
\hline & 04120 & $\begin{array}{l}\text { Ubiquitin mediated } \\
\text { proteolysis }\end{array}$ & 7 & 0.0185 & $\begin{array}{l}\text { ANAPC13, BIRC6, FBXO4, ITCH, KLHL9, } \\
\text { UBE2J1 and UBE2Q1 }\end{array}$ \\
\hline & 04340 & $\begin{array}{l}\text { Hedgehog signaling } \\
\text { pathway }\end{array}$ & 4 & 0.0255 & BMP4, GLI2, STK36 and WNT7B \\
\hline & 05200 & Pathways in cancer & 12 & 0.0300 & $\begin{array}{l}\text { BMP4, DAPK1, FZD2, GLI2, IL6, PLD1, RARB, } \\
\text { STAT1, STK36, TCF7L2, TRAF5 and WNT7B }\end{array}$ \\
\hline
\end{tabular}

$\mathrm{n}$, number of genes enriched in the corresponding pathway; KEGG, Kyoto Encyclopedia of Genes and Genomes.

Additionally, the other upregulated DEG $M A X$ was enriched in the pathways in cancer and identified to be a TAG in the present study. MAX is a member of the basic helix-loop-helix leucine zipper family of TFs and could interact with other family members, including v-myc avian myelocytomatosis viral oncogene homolog (MYC), MAX interactor 1, dimerization protein and mothers against decapentaplegic (MAD), to form homodimers or heterodimers (31). The study by Grandori et al (32) demonstrated that the MYC/MAX/MAD network was comprised of a group of TFs controlling cell cycle differentiation, progression and death. Additionally, Zhu et al (33) reported that levels of MAD1 rapidly decrease upon mitogen stimulation, and this degradation could be regulated by the MAPK pathway. Furthermore, Goel et al (34) reported that DAMTC could induce apoptosis through the mitochondrial pathway by modulating the MAPK pathway. Thus, it was indicated that DAMTC may induce cell apoptosis by upregulating the expression of $M A X$ and modulating the MAPK pathway in lung adenocarcinoma. 


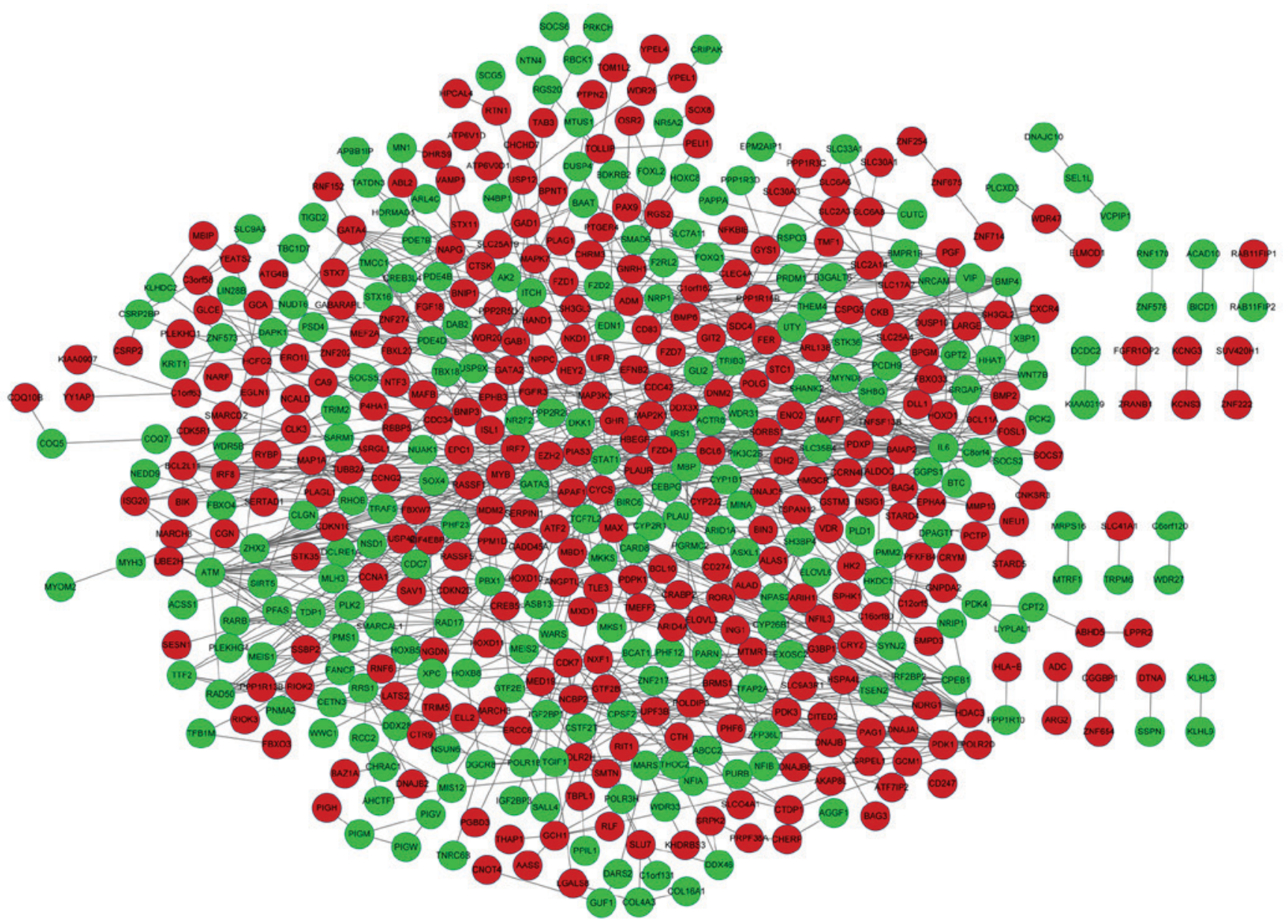

Figure 2. Protein-protein interaction network of differentially-expressed genes. The red nodes indicate upregulated genes and green nodes represent downregulated genes.

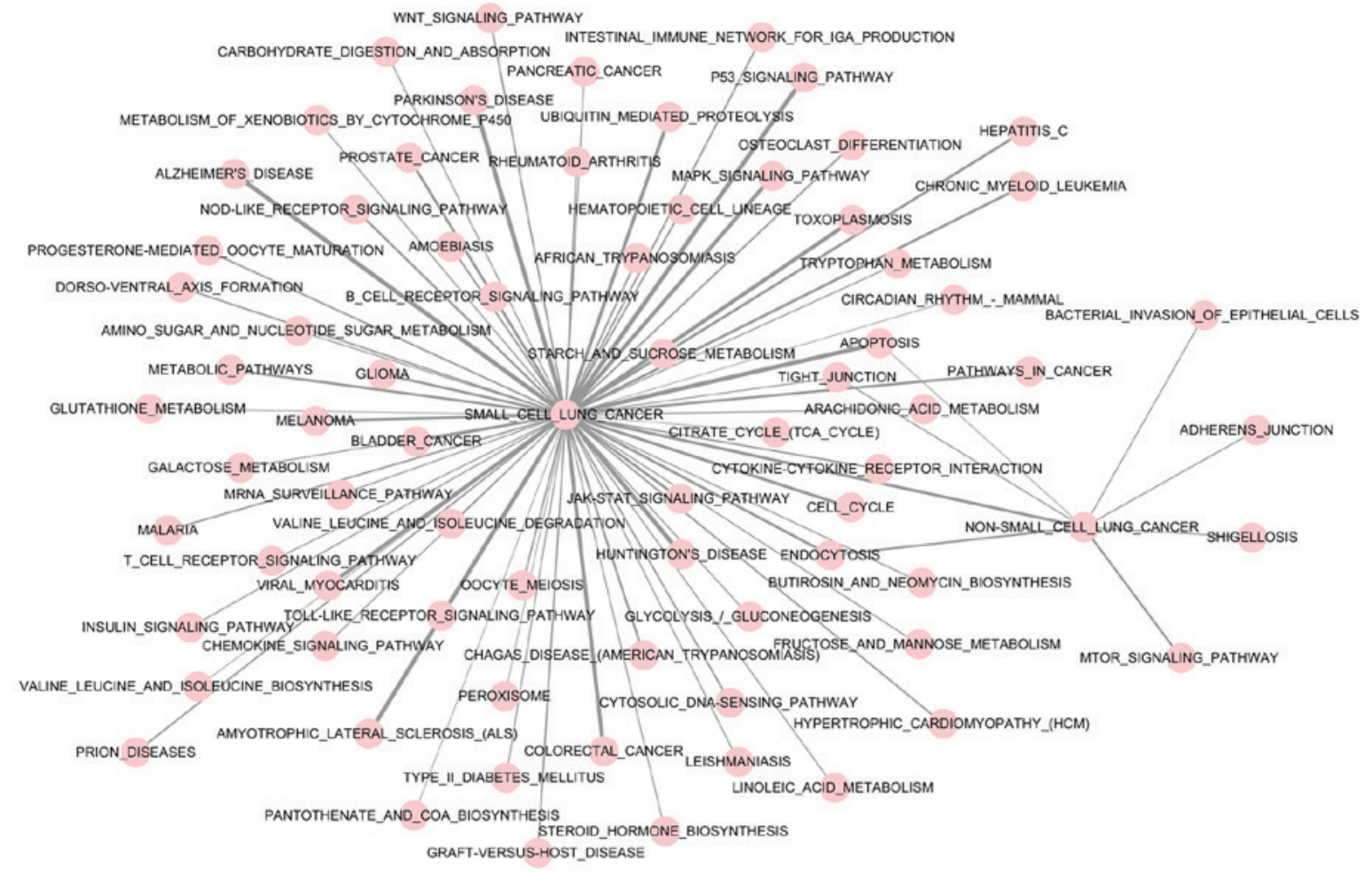

Figure 3. Pathway crosstalk analysis of 7, 8-diacetoxy-4-methylcoumarin-treated lung adenocarcinoma. The pink nodes represent the Kyoto Encyclopedia of Genes and Genomes pathway. The width of solid line indicates the strength of the crosstalk. 
Table III. Functional annotation of differentially-expressed genes in 7,8-diacetoxy-4-methylcoumarin-treated lung cancer groups.

Expression

changes

TF

TSG

Other TAG

\begin{tabular}{llll}
\hline Upregulated & ATF2, BCL6, CDK7, ERCC6, & APAF1, ARID3B, BCL10, BIK, BMP2, & CHRM3, FZD7, LGALS8 \\
& EZH2, FOXD1, GATA2, GATA4, & BRMS1, CAMTA1, CDKN1C, & MAFB, MAP1A, MAX, \\
& GMEB1, GTF2B, HAND1, HDAC3, & CDKN2D, DDX3X, EGLN1, FBXW7, & PIAS3 and RGS2 \\
& HEY2, HOXD10, & HDAC3, ING1, IRF8, LATS2, NDRG1, & \\
& HOXD11, ING1, IRF7, IRF8, IRX5, & PHF6, PLAGL1, PLEKHO1, PPP1R3C, & \\
& ISL1, MAFB, MAFF, MAX, & RASSF1, RASSF5, SLC9A3R1, & \\
& MEF2A, NFIL3, PAX9, PLAGL1, & TMEFF2 and TP53INP1 & \\
& RFX2, RORA, SOX8, TMF1, & & ATM, PMS1, RHOB, \\
& VDR, ZNF10 and ZNF133 & & \\
Downregulated & AKNA, CEBPG, FOXL2, FOXQ1, & ABLIM3, ARID1A, ASXL1, CDH4, & \\
& GATA3, GLI2, GTF2E1, HOXB5, & COL4A3, DAB2, DAPK1,DFNA5, & \\
& HOXB8, HOXC8, MEIS1, MEIS2, & DKK1, KCNRG, KRIT1, MN1, MTUS1, \\
& MSC, NFIA, NFIB,NPAS2, NR2E3, & NRCAM, PLK2, PRDM1, RARB and \\
& NR2F2, NR5A2, PBX1, PRDM1, & TCF7L2 \\
& SMAD6, SOX4, STAT1, TBX18, & & \\
& TCF7L2 and XBP1
\end{tabular}

TF, transcription factor; TSG, tumor suppression genes; TAG, tumor-associated gene.

Furthermore, pathway crosstalk analysis in the present study demonstrated that apoptosis, tight junction and endocytosis had cross-talks with non-small and small lung cancer. Additionally, the present study also revealed that $M D M 2$ and $C D C 42$, which were another two hub genes in the PPI network, were identified to be involved in different pathways, including the p53 signaling and endocytosis pathways. MDM2 encodes a nuclear-localized E3 ubiquitin ligase that mediates ubiquitination of p53 (35). Oren (36) demonstrated that proteins encoded by one or more p53 target genes could serve an essential role in causing p53-mediated apoptosis. This was consistent with the observations by Goel et al (12), demonstrating that DAMTC could induce apoptosis by modulating the p53 pathway in A549 cells. For the other DEG, CDC42 is a small GTPase of the Rho-subfamily, which can regulate signaling pathways that control numerous cellular functions, including cell endocytosis, migration and cell cycle progression (37). Georgiou et al (38) reported that CDC42 could act together with par- 6 family cell polarity regulator and protein kinase $\mathrm{C}$ in the regulation of Arp2/3-mediated endocytosis to control local adherens junction stability, modulating actin filament dynamics (38). Additionally, Mosesson et al (39) revealed that aberrant endocytosis of transmembrane proteins could contribute to malignant transformation. Furthermore, Goel et al (34) demonstrated that DAMTC could inhibit cell motility in A549 cells. Collectively, it was confirmed that DAMTC could induce cancer cell apoptosis by modulating the p53 pathway. In addition, it was indicated that DAMTC may inhibit cell motility through targeting $C D C 42$ and affecting the endocytosis pathway in lung adenocarcinoma.

In conclusion, the present study determined a network of genes, including IL6, MDM2, CDC42 and MAX, targeted by DAMTC that participated in different pathways were involved in the mechanism of DAMTC-treated lung adenocarcinoma.
DAMTC may induce cell apoptosis by targeting IL6 and MAX, which was involved in the MAPK pathway in lung adenocarcinoma. Additionally, DAMTC may inhibit cell motility through targeting CDC42 and affecting the endocytosis pathway in lung adenocarcinoma. These activities may contribute to the anti-carcinogenic action of DAMTC. Due to the relatively small number of samples in the present study, further studies using a larger sample size to validate and determine the role of the potential molecular mechanism identified are required. Ongoing studies may emphasize the potential of DAMTC as an anticancer therapeutic.

\section{Acknowledgements}

Not applicable.

\section{Funding}

Not applicable.

\section{Availability of data and materials}

The datasets used and/or analyzed during the present study are available on reasonable request from the corresponding author.

\section{Authors' contributions}

BW conceived and designed the study. YC and YK acquired the data. XL and HF analyzed and interpreted the data. SZ and YK performed statistical analysis. BW drafted the manuscript. YC and TZ revised the manuscript for important intellectual content. All authors read and approved the final manuscript. 


\section{Ethics approval and consent to participate}

Not applicable.

\section{Patient consent for publication}

Not applicable.

\section{Competing interests}

The authors declare that they have no competing interests.

\section{References}

1. Goel A, Chhabra R, Ahmad S, Prasad A, Parmar V, Ghosh B and Saini N: DAMTC regulates cytoskeletal reorganization and cell motility in human lung adenocarcinoma cell line: An integrated proteomics and transcriptomics approach. Cell Death Dis 3: e402, 2012

2. Siegel R, DeSantis C, Virgo K, Stein K, Mariotto A, Smith T, Cooper D, Gansler T, Lerro C, Fedewa S, et al: Cancer treatment and survivorship statistics, 2012. CA Cancer J Clin 62: 220-241, 2012.

3. Kaisermann MC, Trajman A and Madi K: Evolving features of lung adenocarcinoma in Rio de Janeiro, Brazil. Oncol Rep 8 189-192, 2001

4. Imielinski M, Berger AH, Hammerman PS, Hernandez B, Pugh TJ, Hodis E, Cho J, Suh J, Capelletti M, Sivachenko A, et al: Mapping the hallmarks of lung adenocarcinoma with massively parallel sequencing. Cell 150: 1107-1120, 2012

5. Borges F, Roleira F, Milhazes N, Santana L and Uriarte E: Simple coumarins and analogues in medicinal chemistry: Occurrence, synthesis and biological activity. Curr Med Chem 12: 887-916, 2005.

6. Musa MA, Cooperwood JS and Khan MO: A review of coumarin derivatives in pharmacotherapy of breast cancer. Curr Med Chem 15: 2664-2679, 2008.

7. Kostova I, Raleva S, Genova P and Argirova R: Structure-activity relationships of synthetic coumarins as HIV-1 inhibitors. Bioinorg Chem Appl: 68274, 2006.

8. Kontogiorgis CA and Hadjipavlou-Litina DJ: Synthesis and biological evaluation of novel coumarin derivatives with a 7-azomethine linkage. Bioorg Med Chem Lett 14: 611-614, 2004.

9. Appendino G, Mercalli E, Fuzzati N, Arnoldi L, Stavri M, Gibbons S, Ballero M and Maxia A: Antimycobacterial coumarins from the sardinian giant fennel (Ferula communis). J Nat Prod 67: 2108-2110, 2004.

10. Lacy A and O'Kennedy R: Studies on coumarins and coumarin-related compounds to determine their therapeutic role in the treatment of cancer. Curr Pharm Des 10: 3797-3811, 2004

11. Goel A, Prasad A, Parmar V, Ghosh B and Saini N Antiproliferative effects of 7,8 diacetoxy 4 methyl coumarin and 7, 8 diacetoxy 4 methyl thiocoumarin in human lung adenocarcinoma cell line. Clin Cancer Res 13: C38-C38, 2007.

12. Goel A, Prasad AK, Parmar VS, Ghosh B and Saini N: Apoptogenic effect of 7, 8-diacetoxy-4-methylcoumarin and 7, 8-diacetoxy-4-methylthiocoumarin in human lung adenocarcinoma cell line: Role of NF-kappaB, Akt, ROS and MAP kinase pathway. Chem Biol Interact 179: 363-374, 2009.

13. Smyth GK: Limma: Linear models for microarray data In: Bioinformatics and Computational Biology Solutions Using R and Bioconductor. Gentleman R, Carey VJ, Huber W, Irizarry RA and Dudoit S (eds). Springer, New York, NY, pp397-420, 2005.

14. Reiner-Benaim A: FDR Control by the BH Procedure for two-sided correlated tests with implications to gene expression data analysis. Biom J 49: 107-126, 2007.

15. Benjamini Y and Hochberg Y: Controlling the false discovery rate: A practical and powerful approach to multiple testing. J R Stat Soc Series B (Methodological) 57: 289-300, 1995.

16. Ashburner M, Ball CA, Blake JA, Botstein D, Butler $\mathrm{H}$, Cherry JM, Davis AP, Dolinski K, Dwight SS, Eppig JT, et al: Gene ontology: Tool for the unification of biology. The Gene Ontology Consortium. Nat Genet 25: 25-29, 2000.
17. Huang da W, Sherman BT and Lempicki RA: Systematic and integrative analysis of large gene lists using DAVID bioinformatics resources. Nat Protoc 4: 44-57, 2009.

18. Kanehisa M and Goto S: KEGG: Kyoto encyclopedia of genes and genomes. Nucleic Acids Res 28: 27-30, 2000.

19. Du P, Feng G, Flatow J, Song J, Holko M, Kibbe WA and Lin SM: From disease ontology to disease-ontology lite: Statistical methods to adapt a general-purpose ontology for the test of gene-ontology associations. Bioinformatics 25: i63-i68, 2009.

20. Matys V, Fricke E, Geffers R, Gössling E, Haubrock M, Hehl R, Hornischer K, Karas D, Kel AE, Kel-Margoulis OV, et al: TRANSFAC: Transcriptional regulation, from patterns to profiles. Nucleic Acids Res 31: 374-378, 2003.

21. Köhler S, Bauer S, Horn D and Robinson PN: Walking the interactome for prioritization of candidate disease genes. Am J Hum Genet 82: 949-958, 2008.

22. Franceschini A, Szklarczyk D, Frankild S, Kuhn M, Simonovic M, Roth A, Lin J, Minguez P, Bork P, von Mering C and Jensen LJ: STRING v9. 1: Protein-protein interaction networks, with increased coverage and integration. Nucleic Acids Res 41 (Database Issue): D808-D815, 2013.

23. Von Mering C, Huynen M, Jaeggi D, Schmidt S, Bork P and Snel B: STRING: A database of predicted functional associations between proteins. Nucleic Acids Res 31: 258-261, 2003.

24. Li Y, Agarwal P and Rajagopalan D: A global pathway crosstalk network. Bioinformatics 24: 1442-1447, 2008

25. Liu ZP, Wang Y, Zhang XS and Chen L: Identifying dysfunctional crosstalk of pathways in various regions of Alzheimer's disease brains. BMC Syst Biol 4 (Suppl 2): S11, 2010.

26. Cooper S and Spiro SG: Small cell lung cancer: Treatment review. Respirology 11: 241-248, 2006.

27. Atreya R, Mudter J, Finotto S, Müllberg J, Jostock T, Wirtz S, Schütz M, Bartsch B, Holtmann M, Becker C, et al: Blockade of interleukin 6 trans signaling suppresses T-cell resistance against apoptosis in chronic intestinal inflammation: Evidence in crohn disease and experimental colitis in vivo. Nat Med 6: 583-588, 2000.

28. Hodge DR, Hurt EM and Farrar WL: The role of IL-6 and STAT3 in inflammation and cancer. Eur J Cancer 41: 2502-2512, 2005.

29. Fesik SW: Promoting apoptosis as a strategy for cancer drug discovery. Nat Rev Cancer 5: 876-885, 2005.

30. Nicholson DW: From bench to clinic with apoptosis-based therapeutic agents. Nature 407: 810-816, 2000.

31. Lüscher B: Function and regulation of the transcription factors of the Myc/Max/Mad network. Gene 277: 1-14, 2001.

32. Grandori C, Cowley SM, James LP and Eisenman RN: The Myc/Max/Mad network and the transcriptional control of cell behavior. Annu Rev Cell Dev Biol 16: 653-699, 2000.

33. Zhu J, Blenis J and Yuan J: Activation of PI3K/Akt and MAPK pathways regulates Myc-mediated transcription by phosphorylating and promoting the degradation of Mad1. Proc Natl Acad Sci USA 105: 6584-6589, 2008.

34. Goel A, Chhabra R, Ahmad S, Prasad AK, Parmar VS, Ghosh B and Saini N: DAMTC regulates cytoskeletal reorganization and cell motility in human lung adenocarcinoma cell line: An integrated proteomics and transcriptomics approach. Cell Death Dis 3: e 402, 2012.

35. Stevenson LF, Sparks A, Allende-Vega N, Xirodimas DP, Lane DP and Saville MK: The deubiquitinating enzyme USP2a regulates the p53 pathway by targeting Mdm2. EMBO J 26: 976-986, 2007.

36. Oren M: Decision making by p53: Life, death and cancer. Cell Death Differ 10: 431-442, 2003.

37. Pula G and Poole AW: Critical roles for the actin cytoskeleton and cdc42 in regulating platelet integrin alpha2beta1. Platelets 19: 199-210, 2008

38. Georgiou M, Marinari E, Burden J and Baum B: Cdc42, Par6 and aPKC regulate Arp2/3-mediated endocytosis to control local adherens junction stability. Curr Biol 18: 1631-1638, 2008.

39. Mosesson Y, Mills GB and Yarden Y: Derailed endocytosis: An emerging feature of cancer. Nat Rev Cancer 8: 835-850, 2008.

This work is licensed under a Creative Commons Attribution-NonCommercial-NoDerivatives 4.0 International (CC BY-NC-ND 4.0) License. 